\title{
Protective Effects of Many Citrus Flavonoids on Cartilage Degradation Process
}

\section{Lucia Crascì, Annamaria Panico}

Department of Drug Sciences, Faculty of Pharmacy, University of Catania, Catania, Italy.

Email: luciacrasci@alice.it

Received May $17^{\text {th }}, 2013$; revised June $20^{\text {th }}, 2013$; accepted July $4^{\text {th }}, 2013$

Copyright (C) 2013 Lucia Crascì, Annamaria Panico. This is an open access article distributed under the Creative Commons Attribution License, which permits unrestricted use, distribution, and reproduction in any medium, provided the original work is properly cited.

\begin{abstract}
The objective of this study was to investigate the effects of many citrus flavanones, such as neoeriocitrin, naringin and neohesperidin, in cartilage degradation. Degenerative joint disease involved degradation of joints, including articular cartilage and subchondral bone. When bone surfaces become less well protected by cartilage, bone may be exposed and damaged. The degradation cartilage is mediated by alteration of the balance between anabolic and catabolic processes, changes in proteolytic enzyme activity, mechanical disruption of the cartilage extracellular matrix (ECM), or a combination of these processes. We examine the capability of neoeriocitrin, naringin and neohesperidin, to inhibit metalloproteinase (MMP)-13, collagenase involved in degradation of cartilage matrix components. Also, we assay the flavonoids effect on reducing of Glycosaminoglycans (GAGs) release, and restore Nitric oxide (NO) levels in explant of human articular cartilage. Our results suggest that neoeriocitrin, naringin and neohesperidin are a potential therapeutic agent to protect cartilage tissue.
\end{abstract}

Keywords: Neoeriocitrin; Naringin; Neohesperidin; Metalloproteinases-13; Cartilage Degradation

\section{Introduction}

Osteoarthritis (OA) is a major debilitating disease and affects millions of people all over the world. OA is a slowly progressive disease with multiple etiologies involving biomechanical, biochemical, and genetic factors, all of which may contribute to the OA lesion in cartilage. Age, oxidative stress and inflammation are factors determining the onset and the progression of the disease [1].

The medical attention has turned to means of preventing OA, treating symptoms and slowing its progression. Traditional treatments including non-steroidal anti-inflammatory drugs, or corticosteroids, have had limited success and are often accompanied by significant side-effects preventing long-term use.

Compounds extracted from natural plants or traditional medicinal plants have drawn increasing attention for their clinical efficacy with minimal side effects. Different studies demonstrated that plant-derived phenolic compounds such as flavonoids have antioxidant properties capable of reducing the risk of developing OA [2]. Other study showed that flavonoids were very reactive towards reactive oxygen species (ROS), and they inhibited the activities of many enzymes, such as metalloproteinases (MMPs), nuclear transcription factor kappa B (NF- $\mathrm{KB})$ and also the expression of genes associated in chronic inflammatory disease [3].

Based on these considerations, we evaluated the antidegenerative effects of citrus flavanones especially of naringin, neohesperidin and neoeriocitrin, that are known to exert many biological actions correlated to antioxidant activity, in cartilage degradation process [4-6].

During the degenerative process of the joints, the physical properties of the ECM become impaired and a loss of collagen and aggrecan from cartilage occurs. Pro-inflammatory cytokines, such as interleukin- $1 \beta$ (IL-1 $\beta)$ and other mediators produced by cytokine action, may contribute to this process, accelerating the degradation of cartilage matrix [7]. IL-1 $\beta$ can strikingly upregulate matrix MMPs expression and induce the progression of OA disease [8]. In particular, MMP-13 is considered the major collagenase in OA and has been shown to degrade type II collagen [9]. Moreover, when destructive processes override those of repair, proteoglycans and other related extracellular matrix components of articular cartilage, such as glycosaminoglycans (GAGs), are released, resulting in 
the loss of load bearing properties of cartilage [10].

IL-1 $\beta$ active inflammatory responses producing abnormal levels of Reactive Nitrogen Species (RNS) and Reactive Oxygen Species (ROS); this coud has many adverse consequences, either directly or as a result of increasing radical generation by reacting with $\mathrm{O}_{2}[11]$.

In order to evaluate the anti-degenerative profile, in OA cartilage, of citrus flavanones, neoeriocitrin, naringin and neohesperidin, we assayed their capability to inhibit MMP-13. Also, we demonstrated the ability of the tested flavonoids to prevent IL- $1 \beta$ induced GAGs release and NO production in a model of cartilage degradation.

\section{Materials and Methods}

\subsection{MMP-13 Fluorimetric Assay}

The neoeriocitrin, naringin, neohesperidin were evaluated for their ability to inhibit the hydrolysis of fluorescence-quenched peptide substrate Mca-Pro-Leu-Gly-LeuDpa-Ala-Arg- $\mathrm{NH}_{2}$ (Biomol, Inc.). The MMP-13 assays were performed in $50 \mathrm{mM}$ HEPES buffer containing 5 $\mathrm{mM} \mathrm{CaCl} 2,0.1 \mathrm{mM} \mathrm{ZnCl}_{2}, 0.05 \%$ Brij-35, at pH 7, using $10 \mathrm{nM}$ of proteolytic enzyme (catalytic domains of MMP-13) (Biomol, Inc.) and $350 \mathrm{nM}$ of peptide. The enzyme was incubated at $25^{\circ} \mathrm{C}$ with increasing concentration of the inhibitor and the fluorescence (excitation $\max$

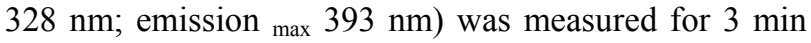
after the addition of the substrate using a Varian Eclipse fluorimeter. Fitting of rates as a function of inhibitor concentration provided $\mathrm{IC}_{50}$ values.

The inhibitor $N$-isobutyl- $N$-[4-methoxyphenylsulfonyl] glycyl hydroxamic acid (Biomol, Inc.) was used as control [12].

\subsection{Human Articular Cartilage Culture}

Human articular cartilage was obtained from patients with femoral neck fractures undergoing replacement surgery. The isolation procedure was conducted under antiseptic conditions. The cartilage was cut into small fragments and carefully washed using Dulbecco's Modified Eagle's Medium (DMEM) culture medium containing $\mathrm{NaHCO}_{3}$. The fragments were set in a sterile 24 well plate, into each containing DMEM (Sigma) phenol red free $(1 \mathrm{~mL})$, fetal bovine serum (FCS, $10 \%)$, glutamine $(10 \mathrm{mM})$, penicillin $\left(100 \mathrm{U} \cdot \mathrm{mL}^{-1}\right)$, streptomycin $(100$ $\left.\mu \mathrm{g} \cdot \mathrm{mL}^{-1}\right)$, gentamycin $\left(50 \mu \mathrm{g} \cdot \mathrm{mL}^{-1}\right)$, and amphotericyn $\mathrm{B}\left(2.5 \mathrm{mg} \cdot \mathrm{mL}^{-1}\right)$. After $24 \mathrm{~h}$ incubation at $37^{\circ} \mathrm{C}$, with $5 \%$ $\mathrm{CO}_{2}$ and $95 \%$ humidified air, the medium was removed and cartilage samples were treated as follows $(n=3$ per group): 1) control medium; 2) IL-1 $\left.\beta\left(10 \mathrm{ng} \cdot \mathrm{mL}^{-1}\right) ; 3\right)$ flavanones $\left(10\right.$ or $\left.50 \mu \mathrm{g} \cdot \mathrm{mL}^{-1}\right)$ combined with IL-1 $\beta(10$ $\left.\mathrm{ng} \cdot \mathrm{mL}^{-1}\right)$. After $120 \mathrm{~h}$ the cartilage culture supernatant was collected for different assays.

\subsection{Determination of Glycosaminoglycans (GAGs)}

The level of GAGs, an index of cartilage damage, was measured by spectrophotometry with a solution of 1,9dimethylmethylene blue at $\lambda=535 \mathrm{~nm}$ using the method of Farndale et al. [13]. The amount of glycosaminoglycans was calculated from a standard curve $(100-500$ $\mu \mathrm{g} \cdot \mathrm{mL}^{-1}$ ) obtained for shark chondroitin sulphate $\mathrm{C}$, derived from shark cartilage.

\subsection{Determination of Nitrite}

Nitrite was determined by adding $100 \mu \mathrm{l}$ of Griess reagent ( $1 \%$ sulphanylamide and $0.1 \%$ naphthylethylenediamine dihydrochloride in $5 \%$ of hydrochloric acid) to $100 \mu \mathrm{l}$ of samples [14]. The optical density at $\lambda=570 \mathrm{~nm}$ was measured using a microtiter plate reader. Nitrite concentrations were calculated by comparison with respective optical densities of standard solutions of sodium nitrite standard curve $(0-120 \mu \mathrm{M})$.

\subsection{Statistical Analysis}

All the present results are means \pm SEM of three experiments performed on quadruplicate samples. The Student $t$ test was used to evaluate the differences between the means of each group. $\mathrm{P}<0.05$ was considered to be statistically significant.

\section{Results}

\subsection{Mmp-13 Inhibition of Neoeriocitrin, Naringin and Neohesperidin}

The neoeriocitrin, naringin and neohesperidin were evaluated for their capability to inhibit MMP-13, collagenase over-expressed in different forms of tissue degenation. The assay was performed using a fluorescencepeptide substrate (Mca-Pro-Leu-Gly-Leu-Dpa-Ala-Arg$\mathrm{NH}_{2}$ ). Table 1 reports the results of the MMP-13 inhibitory activity of tested citrus flavanones. Out of the tested compounds, the neohesperidin proved to be the most potent MMP-13 inhibitor $\left(\mathrm{IC}_{50}=53.50 \mu \mathrm{M}\right)$, followed by naringin $\left(\mathrm{IC}_{50}=157.46 \mu \mathrm{M}\right)$. Instead, the neoeriocitrin was not active.

\subsection{Effect of Neoeriocitrin, Naringin and Neohesperidin on Gags Release}

The neoeriocitrin, naringin and neohesperidin activity on GAGs levels was evaluated at different concentrations $\left(10\right.$ and $\left.50 \mu \mathrm{g} \cdot \mathrm{mL}^{-1}\right)$ in explants of human cartilage, after 120 hours of treatment. IL-1 $\beta$, pro-inflammatory cytokine, was used at a concentration of $10 \mathrm{ng} \cdot \mathrm{mL}^{-1}$. Figure 1 showed that the untreated control samples released, in 
Table 1. In vitro MMP-13 inhibitory activity of neoeriocitrin, naringin, and neohesperidin.

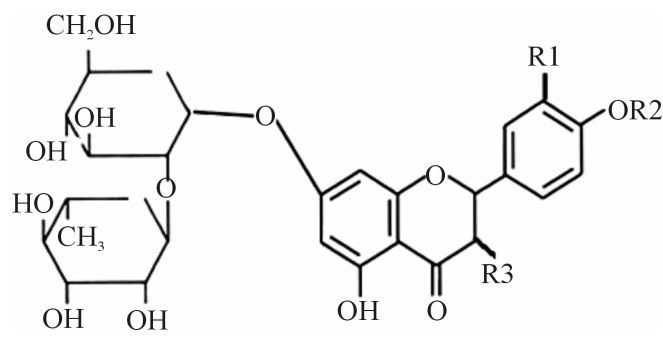

\begin{tabular}{ccccc}
\hline COMPOUNDS & $\mathrm{R} 1$ & $\mathrm{R} 2$ & $\mathrm{R} 3$ & $\mathrm{IC}_{50} \mathrm{MMP}-13(\mu \mathrm{M})$ \\
\hline Neoeriocitrin & $\mathrm{OH}$ & $\mathrm{H}$ & $\mathrm{H}$ & $\mathrm{n} . \mathrm{a}$ \\
Naringin & $\mathrm{H}$ & $\mathrm{H}$ & $\mathrm{H}$ & $157.46 \pm 0.5$ \\
Neohesperidin & $\mathrm{OH}$ & $\mathrm{Me}$ & $\mathrm{H}$ & $53.50 \pm 0.25$
\end{tabular}

n.a. $=$ no activity at $100 \mu \mathrm{M}$. Representative results of at least 3 independent experiments are reported.

culture medium, $620 \pm 0.3 \mu \mathrm{g} \cdot \mathrm{mL}^{-1}$ of GAGs. When the samples were treated with IL-1 $\beta$ the amount of GAGs increases $\left(1.380 \pm 0.8 \mu \mathrm{g} \cdot \mathrm{mL}^{-1}\right)$, all the tested flavonoids, in combination with IL- $1 \beta$, suppressed the IL- $1 \beta$ induced GAGs release from matrix cartilage and nehoesperidin showed the highest activity, at both concentrations used.

\subsection{Effect of Neoeriocitrin, Naringin and Neohesperidin on NO Production}

The results obtained after $120 \mathrm{~h}$, at the concentration of 10 and $50 \mu \mathrm{g} \cdot \mathrm{mL}^{-1}$ of tested flavonoids, are reported in Figure 2. It can be observed that the untreated controls produced a low amount of NO, $(4.2 \pm 0.5 \mu \mathrm{M})$ mainly due to the activity of the constitutive NO synthase. A remarkable increase in the production was observed after the treatment of the sample with IL- $1 \beta$ at concentration of $10 \mathrm{ng} \cdot \mathrm{mL}^{-1}(28 \pm 0.7 \mu \mathrm{M})$. All the tested flavanones, at both concentrations used, when combined with IL-1 $\beta$, exhibited a significant reduction in NO release compared to the samples treated with IL- $1 \beta$.

\section{Discussion}

$\mathrm{OA}$ is a degenerative process of the joints, which is characterized by the progressive destruction and erosion of cartilage. IL-1 $\beta$ has been shown to sustaining cartilage damage by promoting excessive cartilage destruction and by activating the expression of genes for MMPs. MMPs are key enzymes involved in the destruction of articular cartilage in OA [15]. MMP-13 (collagenase-3), a highly expressed collagenolytic MMP in developing bone and cartilage, has been assigned a role in the joint tissue destruction that is a major feature of various forms of human arthritis [16]. Thus, the MMP-13 inhibition reduces

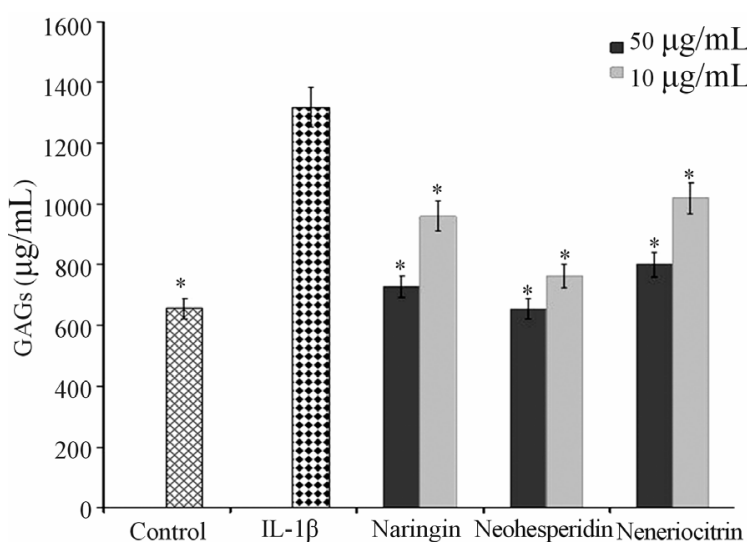

Figure 1. Effect of naringin, neohesperidin and neoeriocitrin flavonoids on GAG release. GAG release $\left(\mu \mathrm{g} \cdot \mathrm{mL}^{-1}\right)$ (means \pm SEM) in the culture medium of articular cartilage, stimulated with IL-1 $\beta, 120$ hrs after the addition of naringin, neohesperidin and neoeriocitrin flavonoids at $1 \mu \mathrm{g} \cdot \mathrm{mL}^{-1}$ and $50 \mu \mathrm{g} \cdot \mathrm{mL}^{-1}$ concentrations. "significantly different from IL-1 $\beta$ treated samples $(P<0.05)$.

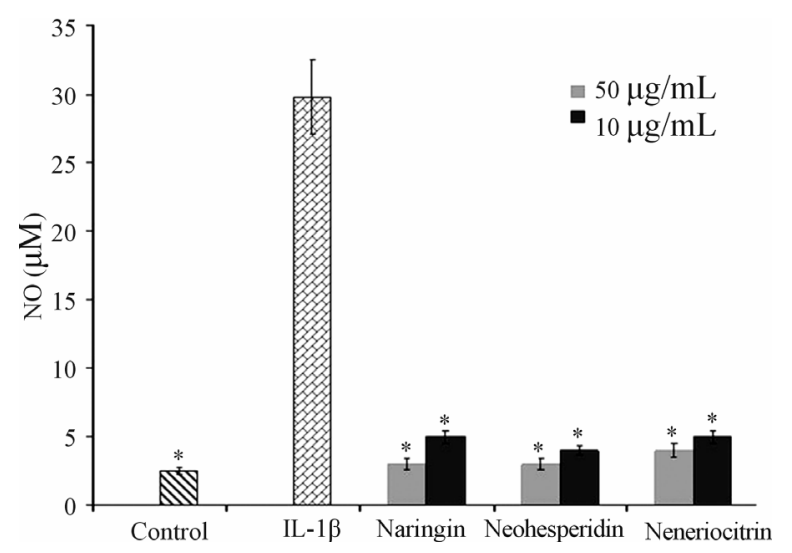

Figure 2. Effect of naringin, neohesperidin and neoeriocitrin flavonoids on NO production. NO production (measured as nitrite, $\mu \mathrm{M})$ (means \pm SEM) in the culture medium of articular cartilage, stimulated with IL-1/, 120 hrs after the addition of naringin, neohesperidin and neoeriocitrin flavonoids at $10 \mu \mathrm{g} \cdot \mathrm{mL}^{-1}$ and $50 \mu \mathrm{g} \cdot \mathrm{mL}^{-1}$ concentrations. "significantly different from IL-1 $\beta$ treated samples $(P<0.05)$.

cartilage degradation associated with the progression of OA in animal models [17]. Studies on the effects of flavonoids on collagen synthesis have progressed to the point of suggesting the potential therapeutic use of these plant derived compounds in the treatment of various medical conditions centered on disrupted collagen metabolism, for example, delayed wound healing, skin aging and OA. Moreover, flavonoids of subclass proanthocyanidins display high affinity for collagen and elastin fiber in addition to preventing their enzymatic hydrolysis by MMPs [18]. Basid on these considerations, the present study was carried out to evaluate the effects of naringin, neohesperidin and neoeriocitrin, many citrus flavanones, on cartilage degradation. The initial experiments estab- 
lished that the tested flavanones inhibit MMP-13 in different manner (Table 1). The highest activity was exhibited by nehoesperidin, and this could be caused by the methoxyl group. It may be hypothesized that the O-methylation, changing hydrophobicity and molecular planarity, could modify the interaction with the MMP-13 pocket site.

A most important event in joint diseases is structural components of cartilage loss. Articular cartilage is composed of an extracellular matrix (ECM), made up largely of aggrecan, which in turn consists of sulfated proteoglycans and collagen. During the development of OA, the physical properties of the ECM become disrupted and a loss of collagen and aggrecan from cartilage occurs [19]. The in vitro effect of neoeriocitrin, naringin and neohesperidin on the GAG release (Figure 1), showed that all the tested compounds reduced the degradation cartilage process. In particular, nehoesperidin are the most potent flavanones, according to the results of inhibition of MMP-13. Whereas, the behavior of naringin and neoeriocitrin could be reasons to the intervention of other mechanisms in the degenerative cartilage process. NO is another critical regulator down stream of IL-1 $\beta$, have long been demonstrated to be catabolic factor that push forward the progression of OA disease by suppressing collagen and proteoglycan synthesis and inducing MMPs synthesis. Inhibition of NO production represents an experimental therapeutic approach in the treatment of inflammatory joint diseases.

In this work we have demonstrated the ability of naringin, neohesperidin and neoeriocitrin to prevent IL- $1 \beta$ induced NO production. Therefore, these interesting in vitro results, indicates a potential modes of action of tested citrus flavonoids on lowering cartilage tissue degradation.

\section{Conclusions}

In conclusion, degenerative cartilage process involved different factors: oxidative stress, imbalance between anabolic and catabolic effects on chondrocyte, inflammation, mechanical stress, etc.

Our results showed that many citrus flavonoids may prevent the degradation of cartilage, acting on different aspects of the pathological process; starting from inhibition of proteolytic enzymes, in particular MMP-13, preventing the GAGs release and reducing the structural and mechanical loss of cartilage. Therefore, naringin, neohesperidin and neoeriocitrin have confirmed their known role as scavengers of free radical species, here expressed as inhibition of the NO production.

\section{REFERENCES}

[1] F. Berenbaum, "Osteoarthritis as an Inflammatory Dis- ease (Osteoarthritis Is Not Osteoarthrosis!)," Osteoarthritis and Cartilage, Vol. 2, No. 1, 2013, pp. 16-21. doi:10.1016/i.joca.2012.11.012

[2] Y. J. Moon, X. Wang and M. E. Morris, "Dietary Flavonoids: Effects on Xenobiotic and Carcinogen Metabolism," Toxicology in Vitro, Vol. 20, No. 2, 2006, pp. 187-210. doi:10.1016/j.tiv.2005.06.048

[3] A. García-Lafuente, E. Guillamón, A. Villares, M. A. Rostagno and J. A. Martínez, "Flavonoids as Anti-Inflammatory Agents: Implications in Cancer and Cardiovascular Disease," Inflammation Research, Vol. 58, No. 9, 2009, pp. 537-552. doi:10.1007/s00011-009-0037-3

[4] G. Mandalari, M. Bennet, A. R. Kirby, R. B. Lo Curto, G. Bisignano, K. W. Waldron and C. B. Faulds, "Enzymatic Hydrolysis of Flavonoids and Pectic Oligosaccharides from Bergamot (Citrus Bergamia Risso) Pee," Journal of Agricultural and Food Chemistry, Vol. 54, No. 21, 2006, pp. 8307-8313. doi:10.1021/jf0615799

[5] L. Di Donna, G. De Luca, F. Mazzotti, A. Napoli, R. Salerno, D. Taverna and G. Sindona, "Statin-Like Principles of Bergamot Fruit (Citrus Bergamia): Isolation of 3-Hydroxymethylglutaryl Flavonoid Glycosides," Journal Natural Products, Vol. 72, No. 7, 2009, pp. 1352-1354. doi:10.1021/np900096w

[6] A. C. E. Graziano, V. Cardile, L. Crascì, S. Caggi, P. Dugo, F. Bonina and A. Panico, "Protective Effects of an Extract from Citrus Bergamia against Inflammatory Injury in Interferon- $\gamma$ and Histamine Exposed Human Keratinocytes," Life Sciences, Vol. 27, No. 90, 2012, pp. 968-974. doi:10.1016/i.lfs.2012.04.043

[7] P.S. Burrage and C.E. Brinckerhoff, "Molecular Targets in Osteoarthritis: Metalloproteinases and Their Inhibitors," Current Drug Targets, Vol. 8, No. 2, 2007, pp. 293-303. doi:10.2174/138945007779940098

[8] H. Takaishi1, T. Kimura, S. Dalal, Y. Okada and J. D’Armiento, "Joint Diseases and Matrix Metalloproteinases: A Role for MMP-13," Current Pharmaceutical Biotechnology, Vol. 9, No. 1, 2008, pp. 47-54. doi: $10.2174 / 138920108783497659$

[9] J. Y. Wang and M. H. Roehrln, "Glycosaminoglycans Are a Potential Cause of Rheumatoid Arthritis," Proceeding of the National Academy of Sciences of the United States of America, Vol. 99, No. 22, 2002, pp. 14362-14367.

[10] A. Panico, R. Maccari, V. Cardile, L. Crascì, S. Ronsisvalle and R. Ottanà, "5-Arylidene-4-Thiazolidinone Derivatives Active as Antidegenerative Agents on Human Chondrocyte Cultures," Medicinal Chemistry, Vol. 9, No. 1, 2013, pp. 84-90. doi:10.2174/157340613804488378

[11] K. E. Armour, R. J. Van't Hof, P. S. Grabowski, D. M. Reid and S. H. Ralston, "Evidence for a Pathogenic Role of Nitric Oxide in Inflammation-Induced Osteoporosis," Journal of Bone and Mineral Research, Vol. 14, No. 12, 1999, pp. 2137-2142. doi:10.1359/jbmr.1999.14.12.2137

[12] I. Bertini, V. Calderone, M. Fragai, A. Giachetti, M. Loconte, C. Luchinat, M. Maletta, C. Nativi and K. J. Yeo, "Exploring the Subtleties of Drug-Receptor Interactions: The Case of Matrix Metalloproteinases," Journal of the American Chemical Society, Vol. 129, No. 9, 2007, pp. 2466-2475. doi:10.1021/ja065156z 
[13] R. W. Farndale, C. A. Sayers and A. J. Barrett, "A Direct Spectrophotometric Microassay for Sulfated Glycosaminoglycans in Cartilage Cultures," Connective Tissue Research, Vol. 9, No. 4, 1982, pp. 247-248. doi:10.3109/03008208209160269

[14] L. C. Green, D. A. Wagner, J. Glogowski, P. L. Skipper, J. S. Wishnok and S. R. Tannenbaum, "Analysis of Nitrate, Nitrite, and [15N] Nitrate in Biological Fluids," Analytical Biochemistry, Vol. 126, No. 1, 1982, pp. 131-138. doi:10.1016/0003-2697(82)90118-X

[15] M. P. Vincenti, I. M. Clark and C. E. Brinckerhoff, "Using Inhibitors of Metalloproteinases to Treat Arthritis. Easier Said than Done?" Arthritis Rheumatoly, Vol. 37, No. 8, 1994, pp. 1115-1126. doi:10.1002/art.1780370802

[16] G. Murphy, V. Kna“uper, S. Atkinson, G. Butler, W. English, M. Hutton, J. Stracke and I. Clark, "Matrix Metalloproteinases in Arthritic Disease," Arthritis Research, Vol.
4, No. 3, 2002 pp. 39-49. doi:10.1186/ar572

[17] P. Vicini, L. Crascì, M. Incerti, S. Ronsisvalle, V. Cardile and A. M. Panico, "Benzisothiazolyliminothiazolidin-4Ones with Chondroprotective Properties: Searching for Potent and Selective Inhibitors of MMP-13," ChemMedChem, Vol. 6, No. 7, 2011, pp. 1199-1202. doi:10.1002/cmdc. 201100223

[18] T. Stipcevic, J. Piljac and D. Vanden Berghe, "Effect of Different Flavonoids on Collagen Synthesis in Human Fibroblasts," Plant Foods for Human Nutrition, Vol. 61, No. 1, 2006, pp. 29-34. doi:10.1007/s11130-006-0006-8

[19] G. Kerckhofs, J. Sainz, M. Wevers, T. Van de Putte and J. Schrooten, "Contrast-Enhanced Nanofocus Computed Tomography Images the Cartilage Subtissue Architecture in Three Dimensions," European Cells \& Materials, Vol. 7, No. 25, 2013, pp. 179-189. 